\title{
Correction to: Compositional characterization of commercial sparkling wines from cv. Ribolla Gialla produced in Friuli Venezia Giulia
}

\author{
Sabrina Voce ${ }^{1} \cdot$ Domen Škrab $^{1,2} \cdot$ Urska Vrhovsek $^{2} \cdot$ Franco Battistutta $^{1} \cdot$ Piergiorgio Comuzzo $^{1} \cdot$ Paolo Sivilotti $^{1}$
}

Published online: 11 November 2020

๑) Springer-Verlag GmbH Germany, part of Springer Nature 2020

\section{Correction to: \\ European Food Research and Technology \\ (2019) 245:2279-2292 \\ https://doi.org/10.1007/s00217-019-03334-9}

In the original publication of the article, Table 4 has been published with an error. The concentration of the last 5 compounds was given in $\mathrm{mg} / \mathrm{L}$ instead of $\mu \mathrm{g} / \mathrm{L}$.

The correct Table 4 is given in this correction.

The original article has been updated.
The original article can be found online at https://doi.org/10.1007/ s00217-019-03334-9.

\section{Piergiorgio Comuzzo piergiorgio.comuzzo@uniud.it \\ $\triangle$ Paolo Sivilotti paolo.sivilotti@uniud.it}

1 Department of Agricultural, Food, Environmental and Animal Sciences, University of Udine, Via delle Scienze 206, 33100 Udine, Italy

2 Department of Food Quality and Nutrition, Edmund Mach Foundation, Research and Innovation Centre, Via Edmund Mach 1, 38010 San Michele all'adige, TN, Italy
Table 4 Tryptophan metabolites detected in Ribolla Gialla commercial sparkling wines

\begin{tabular}{|c|c|c|c|c|}
\hline Compound & Mean \pm SD & MIN & MAX & $\mathrm{CV}$ \\
\hline ILA-SO ${ }_{3} \mathrm{H}$ & $8.53 \pm 10.31$ & 0.66 & 54.20 & 1.21 \\
\hline TOL-SO ${ }_{3} \mathrm{H}$ & $3.73 \pm 2.55$ & 0.23 & 12.49 & 0.68 \\
\hline TYL & $2.55 \pm 0.97$ & 0.93 & 4.79 & 0.38 \\
\hline TYR & $1.13 \pm 0.49$ & 0.08 & 1.98 & 0.43 \\
\hline ILA-GLU- $\mathrm{SO}_{3} \mathrm{H}$ & $0.53 \pm 0.37$ & 0.12 & 1.79 & 0.70 \\
\hline PHE & $0.51 \pm 0.26$ & 0.03 & 0.99 & 0.51 \\
\hline TRP-EE & $0.44 \pm 0.21$ & 0.11 & 0.89 & 0.48 \\
\hline TOL & $0.29 \pm 0.27$ & 0.01 & 1.08 & 0.93 \\
\hline ABA & $0.22 \pm 0.11$ & 0.02 & 0.46 & 0.50 \\
\hline IAA- $\mathrm{SO}_{3} \mathrm{H}$ & $0.20 \pm 0.07$ & 0.13 & 0.39 & 0.35 \\
\hline ABA-GLU & $0.13 \pm 0.05$ & 0.04 & 0.26 & 0.38 \\
\hline TRP & $0.13 \pm 0.10$ & 0.01 & 0.39 & 0.77 \\
\hline ILA-GLU & $0.10 \pm 0.05$ & 0.01 & 0.25 & 0.50 \\
\hline TYR-EE & $0.08 \pm 0.03$ & 0.02 & 0.13 & 0.38 \\
\hline ILA & $0.03 \pm 0.03$ & n.d. & 0.12 & 1.00 \\
\hline KYNA & $0.02 \pm 0.01$ & n.d. & 0.04 & 0.50 \\
\hline AA & $0.01 \pm 0.06$ & n.d. & 0.33 & 6.00 \\
\hline IAA-ASP* & $4.41 \pm 2.87$ & n.d. & 12.35 & 0.65 \\
\hline N-TYR-EE* & $3.86 \pm 2.11$ & n.d. & 9.63 & 0.55 \\
\hline $\mathrm{KYN}^{*}$ & $2.42 \pm 1.98$ & n.d. & 9.24 & 0.82 \\
\hline IAA* & $1.75 \pm 1.63$ & n.d. & 8.84 & 0.93 \\
\hline N-TRP-EE* & $1.08 \pm 0.81$ & n.d. & 3.47 & 0.75 \\
\hline
\end{tabular}

Concentrations are expressed in $\mathrm{mg} \mathrm{L}^{-1}$ or, where indicated, in $\mu \mathrm{g} / \mathrm{L}\left(^{*}\right)$ ILA-SO $\mathrm{S}_{3} \mathrm{H}$ indole-lactic acid-2-sulfonate, $\mathrm{TOL}-\mathrm{SO}_{3} \mathrm{H}$ tryptophol-2-sulfonate, $T Y L$ tryptophol, TYR tyrosine, ILA-GLU-SO${ }_{3} H$ sulfonated indole3-lactic acid, $P H E$ phenylalanine, TRP-EE tryptophan-ethyl ester, TOL tryptophol, $A B A$ abscisic acid, $I A A-\mathrm{SO}_{3} \mathrm{H}$ sulfonated indole 3-acetic acid, $A B A-G L U$ glucoside of abscisic acid, TRP tryptophan, ILA-GLU indole 3-lactic acid glucoside, TYR-EE tyrosine-ethyl ester, ILA indole 3-lactic acid, KYNA kynurenic acid, $A A$ anthranilic acid, IAA-ASP indole 3-acetic acid conjugate with aspartic acid, $N$-TYR-EE $N$-acetyl-tyrosine-ethyl ester, $K Y N$ kynurenine, IAA indole 3-acetic acid, $N$-TRP-EE $N$-acetyltryptophan-ethyl ester, $S D$ standard deviation, $M I N$ minimum value, $M A X$ maximum value, $C V$ coefficient of variation, $n . d$. not detected

Publisher's Note Springer Nature remains neutral with regard to jurisdictional claims in published maps and institutional affiliations. 